\title{
Webinarreihe: Evidenzbasierung im Globalen Lernen
}

Im Mai und Juni 2021 veranstaltete der Lehrstuhl „Allgemeine Pädagogik“ der Otto-Friedrich-Universität Bamberg in enger Zusammenarbeit mit Engagement Global eine breit ausgeschriebene Reihe von Onlineseminaren zur Evidenzbasierung im Globalen Lernen. Diesem Angebot ging die Erfahrung voraus, dass online angebotene Veranstaltungen neben Beschränkungen auch den Kreis möglicher Teilnehmerinnen und Teilnehmer erweitern, und gerade das Gespräch über regionale Grenzen hinweg und über solche zwischen verschiedenen Stakeholdern erleichtern. Ziel war es, forschungsbasierte Erkenntnisse einem breiteren Kreis zugänglich zu machen und mit den Teilnehmenden im Hinblick auf ihre Arbeitsfelder zu diskutieren.

Den Auftakt bildete ein zweistündiges Webinar „Lernen in globalen Partnerschaften - empirische Ergebnisse und Anregungen für evidenzbasierte Praxis“, an welchem mehr als vierzig Personen aus Deutschland, Österreich, Belgien und Uruguay teilnahmen. Es waren Menschen aus der Bildungsverwaltung, Promotorinnen des Globalen Lernens, Vertreter/-innen verschiedener Netzwerke von den UNSECO-Schulen bis zu den ehemaligen Weltwärtsfreiwilligen, Lehrkräfte und Engagierte aus kleineren Nichtregierungsorganisationen.

Annette Scheunpflug bot in ihrem Vortrag „Lernen in und durch Partnerschaften - empirische Ergebnisse und Anregungen für evidenzbasierte Praxis“ reichhaltige Impulse. $\mathrm{Zu}$ nächst führte sie in die Herausforderung, weltgesellschaftliche Deutungsmuster zu lernen, ein, indem sie von Kennzeichen für global reflektiertes soziales Lernen ausgehend Qualitätsstandards für Begegnungen aufzeigt. Sie liegen unter anderen im einbezogenen Zeithorizont längerfristiger Beziehungen, der Fokussierung auf ein gemeinsames Thema und der Eröffnung vielfältiger Mitgestaltungsmöglichkeiten für die Beteiligten. In einem zweiten Teil „Empirische Spuren“ stellte sie neben Metaanalysen systematische Auswertung aus Projekten im eigenen Forschungskontext. Drei Typen lassen sich für ein weltgesellschaftliches Verständnis identifizieren, deren weltgesellschaftliches Verständnis sich in Bezug auf die Wahrnehmung des Gegenübers, auf Differenzsetzungen, den Umgang mit Komplexität und die Motivation von Handlungen unterscheiden lassen.

Ein Typus ist der der Nahraumorientierung, der in einem asymmetrischen Verhältnis zum Gegenüber steht, eine Addition von verschiedenen Nahräumen in der Nähe und der geschiedenen Ferne denkt und hierbei der Komplexitätsüberforderung durch ein Insistieren auf Authentizität begegnet. Handlungen sind im Modus der Hilfe verankert. Der Typus der Gemeinschaftsorientierung identifiziert sein Gegenüber über Gemeinschaft, setzt auf Zugehörigkeit und realisiert die Komplexitätsreduzierung über Identifikation. Für diesen Typus stellt Solidarität die Motivation von Handlungen dar. Ein dritter Typus hat ein Verständnis von Welt als abstrakten sozialen Raum: Das Gegenüber ist auf Augenhöhe verortet, Differenzen werden als Ordnungsstrukturen gedacht, Komplexität metareflexiv eingeholt und die Handlungen durch globale Teilhabe motiviert. Besonders interessant ist, dass diese drei Typen keiner Sukzession folgen, dass es sich abzeichnet, dass Partizipationserfahrungen für die Typenausprägung relevant sind und eine angeleitete Selbstreflexivität zur Ausgestaltung des dritten Typus förderlich ist. Einige Schlussfolgerungen für evidenzbasiertes globales Lernen in Begegnungen lassen sich so ziehen, beispielsweise der gemeinsamen Arbeit an etwas Drittem Vorrang zu gewähren, einen reflektieren Umgang mit Kulturalität zu pflegen und die lokale Vielfalt sichtbar zu machen.

Einer kurzen Diskussion des Vortrags folgten Arbeitsgruppen, in denen die Teilnehmenden in Gruppen ähnlicher Arbeitskontexte gemeinsam an Verbindungen zum eigenen Erfahrungs- und Arbeitsfeld arbeiteten und Ideen für die Umsetzung in die eigene Praxis arbeiteten. Eine reichhaltige Rückmelderunde im Plenum zeigte, dass die Impulse aus der Forschung viele Anschlussmöglichkeiten boten und welche hohe Bedeutung die Teilnehmenden den Austausch jenseits des eigenen Kontextes beimessen. Der Vortrag von Annette Scheunpflug ist abrufbar unter: https://gene.app.box.com/s/tgwzgn q8ecncowobbtokdma61 y5qhk8j

Mehr Informationen zu den weiteren Veranstaltungen der Seminarreihe sind unter https:/www.uni-bamberg.de/allgpaed/forschung/projekte/evidenzbasierung-im-globalen-ler nen/ abrufbar, u.a. auch die Podcasts und Folien der Vorträge.

Susanne Timm

Otto-Friedrich-Universität Bamberg doi.org/10.31244/zep.2021.03.10 